\title{
Incorporation of retinoic acid releasing microspheres into pluripotent stem cell aggregates for inducing neuronal differentiation
}

Jose Carlos Gomez ${ }^{\mathrm{a}}$, John M. Edgar ${ }^{\mathrm{b}}$, Andrew M. Agbay ${ }^{\mathrm{c}}$, Emma Bibault ${ }^{\mathrm{b}}$, Amy Montgomery ${ }^{\mathrm{a}}$, Nima Khadem Mohtaram ${ }^{\mathrm{a}}$,Stephanie M. Willerth ${ }^{\mathrm{a}, \mathrm{c}, \mathrm{d}^{*}}$

a. Department of Mechanical Engineering, University of Victoria, PO Box 1700 Stn CSC, Victoria, BC V8W 2 Y2 Canada

b. Department of Biomedical Engineering, University of Victoria, PO Box 1700 Stn CSC, Victoria, BC V8W 2Y2 Canada

c. Division of Medical Sciences, University of Victoria, PO Box 1700 Stn CSC, Victoria, BC V8W 2Y2 Canada

d. International Collaboration on Repair Discoveries, 818 W 10th Ave, Vancouver, BC V5Z 1M9, Canada

*To whom all correspondence should be addressed.

Contact Information for Dr. Willerth:

Address: PO Box 1700 Stn CSC, Victoria, BC V8W 2Y2 Canada

Email: willerth@uvic.ca

Phone number: (250) 721-7303

Fax Number: (250) 721-6051 


\begin{abstract}
Pluripotent stem cells (PSCs) can form any specialized cell type found in the body making them an excellent tool for regenerative medicine applications. Directed differentiation of PSCs into specific phenotypes can be accomplished by introducing specific chemical cues such as the small molecule retinoic acid (RA). Expressed in the developing nervous system, RA can induce differentiation of PSCs into neural phenotypes including neurons. In this study, we encapsulated all-trans RA within poly ( $\varepsilon$-caprolactone) (PCL) microspheres to generate controlled morphogen release over 28 days. RA/PCL microspheres less than $\sim 10 \mu \mathrm{m}$ in diameter were readily incorporated within the interstitial sites of human induced pluripotent stem cell (hiPSC) aggregates. After 5 days of culture, the microspheres did not induce cytotoxic effects and the hiPSC aggregates containing microspheres showed a decrease in the pluripotency marker SSEA4. After 7 days of culture on laminin surfaces, aggregates expressed the neuronal marker TUJ1 and displayed extended neurite outgrowth. This approach provides consistent RA delivery throughout the aggregate and could be an effective strategy for differentiating cells in vivo. Overall, our results demonstrate that it is possible to combine hiPSC aggregates with RA/PCL microspheres for neural tissue engineering applications.
\end{abstract}

Keywords: controlled release, drug delivery, neural tissue engineering, poly( $\varepsilon$-caprolactone), embryoid body 


\section{Biography}

Dr. Stephanie Willerth currently holds a Canada Research Chair in Biomedical Engineering at the University of Victoria where she is dually appointed in the Department of Mechanical Engineering and Division of Medical Sciences. Her interdisciplinary research group investigates how to engineer neural tissue by combining pluripotent stem cells, controlled drug delivery and biomaterial scaffolds. In 2014, she was named a "Star in Global Health" by Grand Challenges Canada. She is an active member in the International Collaboration on Repair Discoveries (ICORD), a B.C. based organizations committed to finding long term treatment for the repair of spinal cord injuries. Before accepting her faculty position, Dr. Willerth completed a National Institutes of Health sponsored post-doctoral fellowship at the University of California-Berkeley and graduate studies at Washington University. She received undergraduate degrees in Biology and Chemical Engineering from the Massachusetts Institute of Technology. Her inspiration for this work was the 2014 American League Champions, the Kansas City Royals. 


\section{Introduction}

Pluripotent stem cells (PSCs) are primary cells capable of differentiating into any specialized cell-type found in the body as well as self-renewal, both desirable properties when developing cell therapies. ${ }^{14,17,39,47}$ PSCs can be divided into two main categories, embryonic stem cells (ESCs) and induced pluripotent stem cells (iPSCs). ${ }^{6}$ ESCs are isolated from the inner mass of blastocysts and were first derived from mouse embryos followed by isolation from human embryos. 3, 6, 12, 16, 42 iPSCs are adult somatic cells genetically reprogrammed into an embryonic stem cell-like state. The generation of mouse iPSCs (miPSCs) was first reported in 2006 and quickly followed by the production of human iPSCs (hiPSCs) in $2007 .{ }^{40,41}$ With this latest discovery, iPSC technology provides an excellent tool for the development of personalized tissues for regenerative medicine applications. ${ }^{11}$

PSCs are often cultured as 3D spheroid aggregates called embryoid bodies (EBs) to induce differentiation. ${ }^{42}$ EBs show behavior akin to gastrulation as the PSCs spontaneously differentiate and rearrange into defined germ layers. ${ }^{43}$ EB formation serves as an initial assay of PSC pluripotency. It is also the first step in the production of neural progenitor cells (NPCs), cells committed to the neural lineage. EB composition can be altered towards a preferred germ layer by introducing chemical cues or changing the physical properties of the EB. ${ }^{5,31,48,52}$ Physical properties include EB size and morphology as well as the number of cells, which can be tailored during the formation process. ${ }^{13,36}$ Additionally, EB-based differentiation could serve as a scalable solution towards mass production of specialized cells. ${ }^{8}$

Various chemical cues have been shown to influence PSC differentiation into NPCs. ${ }^{38}$ Retinoic acid (RA), a small morphogenic molecule, can be used to induce differentiation of ESCs and iPSCs into motor neurons. ${ }^{4,29,34,35}$ Morphogens, like RA, are typically administered in 
solution with culture media where achieving a uniform distribution is important for predictable and consistent differentiation of PSC aggregates. A model for the diffusion of small molecules such as RA into the interior of EBs suggests that cell consumption rates, aggregate diameter, and cell density are important factors during PSC differentiation. ${ }^{44}$ High consumption rates can lead to low concentrations within the aggregate, which in turn can result in nonhomogeneous differentiation. ${ }^{25,}{ }^{37,}{ }^{44}$ Morphological differences have been observed in mouse ESC (mESC) EBs when diffusion constraints were bypassed through microparticle delivery of small molecules. ${ }^{9}$

Furthermore, while the use of soluble factors is convenient for in vitro differentiation, such strategies cannot be readily translated for in vivo applications. For instance, in neurological disorders such as spinal cord injury (SCI), the injury site contains cues that influence stem cell survival and differentiation. Specifically, the acute phase of SCI is characterized by an inflammatory response that protects surviving tissue by forming a cystic cavity surrounded by a glial scar. ${ }^{19,49,53}$ This inflammatory response severely affects the survival of transplanted NPCs through its secondary injury mechanisms, such as activated macrophages secreting tumor necrosis factor- $\alpha$ (TNF- $\alpha$ ) and interleukin-1 $\beta$ (IL-1 $\beta$ ) that result in death of host oligodendrocytes. ${ }^{33,53}$ In addition to cues present due to secondary injury, grafted NPCs are also subjected to other inhibitory signals such as chondroitin sulfate proteoglycans (CSPGs) produced by reactive astrocytes that surround the injury site. ${ }^{49}$ This harsh environment can cause the differentiation of NPCs into astrocytes, potentially resulting in a painful condition known as allodynia. $^{20,26,45}$ Thus, it is critical to develop strategies for promoting NPC differentiation that overcome these environmental factors present at the injury site. 
To deliver small morphogenic molecules for in vivo applications, these molecules can be encapsulated within biomaterial-based microspheres for sustained release. This approach enables localized delivery of hydrophobic molecules throughout PSC aggregates to promote homogenous differentiation. Other groups have shown that microspheres manufactured from variety of biodegradable polymers can be used to deliver morphogens throughout mESC aggregates. ${ }^{7-10}$ However, the effects of incorporating drug-releasing microspheres into hiPSC aggregates have not been investigated. Moreover, such a strategy has not been used to generate neural tissue by combining hiPSC aggregates with morphogen releasing microspheres.

A number of polymers, such as poly(lactic-co-glycolic acid) (PLGA), have been widely used to produce microspheres due to their biodegradability and low cytotoxicity. ${ }^{2,}{ }^{50}$ However, the mass production of morphogen loaded microspheres from PLGA is prohibitive due to its high cost per milligram. Accordingly, there has been a renewed interest in poly( $\varepsilon$-caprolactone) (PCL) as a low cost alternative for fabricating scaffolds and microspheres. ${ }^{1,} 31,51$ PCL has a biodegradation rate that can extend well over a year, a desirable feature for long-term applications. ${ }^{51}$ These properties offer a compelling case for using PCL in neural tissue engineering where the differentiation process can take up to 150 days. $^{21,39,46}$

In this study, we encapsulated different concentrations of RA within PCL microspheres using a single emulsion technique and characterized the resulting size distribution and release of RA over a 28 day time course. Next, PCL microspheres were successfully incorporated into hiPSC aggregates, which remained intact after 5 days of culture with no cytotoxic effects observed. We measured the expression levels of pluripotency marker SSEA-4 in hiPSCmicrosphere aggregates at Day 5. hiPSC aggregates containing RA releasing microsphere had decreased SSEA-4 expression suggesting that the controlled release of RA induced the 
differentiation of hiPSCs compared to controls. Finally, we showed that after an additional 7 days of culture, these hiPSC-derived aggregates containing RA releasing microspheres could differentiate into neurons. This work shows that PCL microspheres are a viable option for sustained morphogen delivery within hiPSC aggregates.

\section{Materials and methods}

\section{Microsphere fabrication and sterilization}

Microspheres were fabricated by an oil-water $(\mathrm{o} / \mathrm{w})$ single emulsion as previously described. ${ }^{23}$ Briefly, $500 \mathrm{mg}$ of poly ( $\varepsilon$-caprolactone) (PCL) (Mn 45,000; Sigma) were dissolved in $3 \mathrm{ml}$ of dichloromethane (DCM) (reagent/ACS grade; VWR International) and mixed in a stirring hotplate (Corning PC-420). $2 \mathrm{mg}$ and $15 \mathrm{mg}$ of retinoic acid (RA) (All-trans, $\geq 98 \%$ HPLC, powder; Sigma) were dissolved into the solution for an RA loading of $4 \mu \mathrm{g} / \mathrm{mg}$ and $30 \mu \mathrm{g} / \mathrm{mg}$ (w/w, RA/PCL) respectively. No RA was used for the fabrication of unloaded microspheres. $3 \mathrm{ml}$ of $100 \%$ ethanol (Commercial Alcohols) were added to the solution before it was removed from the stirrer. $3 \mathrm{ml}$ of $2 \%(\mathrm{w} / \mathrm{v})$ poly (vinyl alcohol) (PVA) (Mw 13,000-23,000, 87-89\% hydrolyzed; Sigma) solution were slowly added with care not to disrupt the boundary layer. The solution was then emulsified on a vortex mixer (Fisher Scientific) for 12 seconds and immediately added to $100 \mathrm{ml}$ of $0.3 \%(\mathrm{w} / \mathrm{v})$ PVA solution at $35^{\circ} \mathrm{C}$. The solution was stirred at $500 \mathrm{rpm}$ for 4 hours to evaporate the organic solvent. The unloaded, 4 and $30 \mu \mathrm{g} / \mathrm{mg}$ microspheres were collected by centrifugation at $4000 \mathrm{rpm}$ (Eppendorf 5810R) and washed with $\mathrm{dH}_{2} \mathrm{O}$ to remove traces of PVA. Microspheres were then frozen, lyophilized for $24-36$ hours, and stored at $-20^{\circ} \mathrm{C}$ until use. Before incorporation with hiPSC aggregates, microspheres were sterilized using air-plasma (Harrick PDC-32G) on low power for 30 seconds. 


\section{Microsphere characterization}

The size and surface morphology of microspheres was characterized using a Hitachi S4800 FE scanning electron microscope (SEM) as previously described. ${ }^{1}$ Microspheres were suspended in a small volume of ethanol and dispersed over SEM stubs. After the ethanol evaporated, the stubs were sputter coated with gold-palladium using an Anatech Hummer VI sputter coater. Microsphere diameters were quantified using the Quartz-PCI Image Management Systems ${ }^{\circledR}$ software. The resulting distribution of microsphere diameters was plotted as a histogram with bin sizes equal to $1 \mu \mathrm{m}$ intervals between the smallest to the largest diameter observed for each batch. A kernel density estimator function from the R statistical programming language was used to estimate the probability density function of each histogram. Polynomial regression was used to best fit the density function. The population mean, or expected microsphere diameter, is the first moment of the density function, and variance is equal to the second central moment of density function.

\section{Characterization of $\mathrm{RA}$ release from microspheres}

RA concentrations in the microspheres were determined by dissolving $10 \mathrm{mg}$ of 4 and 30 $\mu \mathrm{g} / \mathrm{mg}$ microspheres in DCM. The PCL was then precipitated out of solution with $100 \%$ ethanol and removed by centrifugation. The light absorbance of the supernatant was measured at $354 \mathrm{~nm}$ using an Infinite M200Pro plate reader with the RA concentration being determined using a standard curve. The corresponding encapsulation efficiencies were calculated using the following equation; the recovered RA corresponds to the amount obtained from dissolved microspheres, and the total RA corresponds to the amount initially dissolved in $500 \mathrm{mg}$ of PCL. 


$$
\left[\frac{\text { Recovered } R A(\mu g)}{\text { Total RA used }(\mu g)}\right] \times 100 \%
$$

Release studies were performed as follows. $10 \mathrm{mg}$ of 4 and $30 \mu \mathrm{g} / \mathrm{mg}$ microspheres were suspended in $1 \mathrm{ml}$ of phosphate buffer saline (PBS) (Life Technologies) in a conical vial. The vials were then placed on a microplate shaker (VWR Microplate Shaker) and incubated at $37^{\circ} \mathrm{C}$. The PBS wash was replaced every 2 days. Multiple vials were labeled for retrieval at a predetermined schedule: day $2,4,8,12,16,20,24$, and 28. PBS was removed during retrieval and microspheres were washed with distilled water, freeze dried, and weighed. Once dry, microspheres were dissolved in DCM and the PCL was precipitated with $100 \%$ ethanol. The solution absorbance was measured at $354 \mathrm{~nm}$ and the RA concentration was determined as previously described. The quantity of RA released was calculated by subtracting the RA remaining in the microspheres from the RA present in day 0 microspheres.

\section{Pluripotent stem cell culture}

Figure 1 shows a schematic of the microsphere incorporation into the hiPSC aggregates. Undifferentiated mESCs (R1, Nagy Lab) were cultured on feeder layers as previously described. ${ }^{32}$ Undifferentiated hiPSCs (iPS(Foreskin)-1, Lot 1-DL-01, WiCell) were maintained on Vitronectin XFTM (STEMCELL Technologies)-coated 6 well plates in TeSR ${ }^{\text {TM}^{\mathrm{T}}}{ }^{\mathrm{E}} 8^{\mathrm{TM}}{ }$ media (STEMCELL Technologies) as previously described. ${ }^{31}$ hiPSCs were dissociated using ReLeSR $^{\text {TM }}$ (STEMCELL Technologies) and uniform aggregates were formed by adding a single cell suspension of $1 \times 10^{6}$ hiPSCs in AggreWell ${ }^{\mathrm{TM}} 800$ inserts (STEMCELL Technologies). hiPSC-microsphere aggregates were formed by resuspending $0.5 \mathrm{mg}$ of microspheres in a small volume ( $<100 \mu \mathrm{l})$ of neural induction medium (NIM) (STEMCELL Technologies), added to each AggreWell ${ }^{\mathrm{TM}} 800$ insert, and centrifuged for 5 minutes at $100 \mathrm{x} \mathrm{g}$ to deposit the cells and 
microspheres. hiPSC, and hiPSC-microsphere aggregates were maintained on AggreWell ${ }^{\mathrm{TM}} 800$ inserts in $2 \mathrm{ml}$ of NIM for 5 days with daily media changes. On day 5, aggregates were dislodged by gentle pipetting and transferred to a poly-L-ornithine (PLO)/laminin-coated (Sigma) 24 well plate. $500 \mathrm{nM}$ of RA was added to the media of a positive control group that did not contain microspheres.

\section{Flow cytometry}

On day 5, cell viability and SSEA-4 marker expression in hiPSC aggregates were assessed using flow cytometry as previously described. ${ }^{32}$ Briefly, aggregates were removed from suspension culture, rinsed twice with PBS, and dissociated enzymatically using $0.05 \%$ trypsinEDTA (Life Technologies) for 20 minutes at $37{ }^{\circ} \mathrm{C}$. The Guava ViaCount reagent (Millipore) was used to measure cell density and viability. Cell suspensions were diluted to 1:10 in Guava ViaCount reagent and incubated for 5 minutes at room temperature protected from light. SSEA-4 expression was measured using the Human/Mouse Pluripotent Stem Cell Multi-Color Flow

Cytometry Kit (R\&D Systems). Single cell suspensions were diluted to $1 \times 10^{6}$ cells per $\mathrm{ml}$ in Fixation Buffer and incubated for 20 minutes at room temperature before being centrifuged into a pellet and re-suspended in Permeabilization Buffer. A single SSEA-4 marker or isotype control was added to the Permeabilization Buffer. Cells were incubated for 1 hour at $4^{\circ} \mathrm{C}$, rinsed and resuspended in 1X Assay Buffer. All data was collected using a Guava EasyCyte HT (Millipore) flow cytometer.

\section{Immunocytochemistry}

On Day 12 of differentiation, hiPSC aggregates were prepared for immunocytochemistry as previously described. ${ }^{32}$ Briefly, the aggregates were fixed with $10 \%$ formalin (Sigma) for 1 
hour at room temperature, permeabilized using 0.1\% Triton-X100 (Sigma) in PBS for 45 minutes at $4{ }^{\circ} \mathrm{C}$, and blocked using $5 \%$ normal goat serum (NGS) for 2 hours at $4{ }^{\circ} \mathrm{C}$. They were then incubated overnight with a 1:500 dilution of TUJ1 primary antibody (anti-III-beta-tubulin; Millipore) followed by three rinses with PBS and a 1:200 dilution of $\operatorname{IgG}(\mathrm{H}+\mathrm{L})$ secondary antibody (AlexaFluor 488 goat anti-mouse; Life Technologies) for 4 hours at room temperature in the absence of light. Samples were rinsed three times to remove unconjugated secondary antibody and counterstained with DAPI (Invitrogen). Images were made using a Leica DMI3000B inverted microscope, Lumen Dynamics X-Cite® 120Q LED fluorescence light source, and QImaging camera and software.

\section{Quantitative analysis of neural aggregate morphology}

Immunostained hiPSC aggregates were imaged with an IncuCyte $\mathrm{ZOOM}{ }^{\circledR}$ live-cell imaging system (Essen BioScience, Ann Arbor, MI). Morphological metrics were quantified using the IncuCyte ZOOM® Software (2015A). Masking and analysis of green channel fluorescence (TUJ1 positive) images for neurite length and branch points were done by the NeuroTrack $^{\mathrm{TM}}$ software module while EB area analysis was done using a Basic Analyzer processing definition. Quantification of the metrics was calculated from 74 separate images between two wells per treatment group. The resulting data were normalized by the number of aggregates present in each well.

\section{SEM imaging of hiPSC aggregates}

The morphology of human cells and the distribution of microspheres within the hiPSC aggregates were analyzed by SEM after 12 days of differentiation. Samples were fixed using $2.5 \%$ glutaraldehyde (Sigma) for 30 minutes, rinsed three times over 15 minutes with $0.1 \mathrm{M}$ 
sodium cacodylate buffer ( $\mathrm{pH} 7.4$; Sigma), then fixed in $1 \%$ osmium tetroxide $\left(\mathrm{OsO}_{4}\right.$; Electron Microscopy Lab, University of Victoria) for 30 minutes. Samples were then rinsed with $\mathrm{dH}_{2} \mathrm{O}$ three times over 15 minutes and dehydrated using a graded series of ethanol. One 5-minute rinse each with $30 \%, 50 \%, 70 \%$, and $90 \%$ ethanol was followed by three 5-minute rinses with $100 \%$ ethanol. The samples were then critically point dried, mounted to SEM stubs, and gold-palladium sputter coated before SEM.

\section{Statistical Analysis}

RA encapsulation efficiencies are reported as mean \pm standard deviation $(n=6)$. Release studies are reported as mean \pm standard error $(\mathrm{n}=3)$. Flow cytometry results are reported as mean \pm standard deviation $(\mathrm{n}=3)$. Statistical significance was determined with standard t-test analysis using the $\mathrm{R}$ statistical programming language. * indicates $\mathrm{p}<0.05$.

\section{Results}

\section{Microsphere fabrication and characterization}

Surface morphology and size distribution were determined to assess microsphere fabrication. SEM images of the microspheres showed a smooth round surface with some clustering [Fig. 2 (A - C)]. 4 and $30 \mu \mathrm{g} / \mathrm{mg}$ microspheres are statistically similar in size with expected diameters of $3.59 \pm 2.48 \mu \mathrm{m}$, and $3.41 \pm 1.60 \mu \mathrm{m}$ respectively, while the unloaded microspheres are slightly smaller with an expected diameter of $2.53 \pm 1.65 \mu \mathrm{m}$ [Fig. 2 (D - F)]. Encapsulation efficiencies for the different loading concentrations of RA are shown in Table 1. Despite the differences in the quantity of RA encapsulated in each sample group, all microspheres had approximately the same encapsulation efficiency $(\sim 60 \%)$. Others have reported 
encapsulation efficiencies with different polymers that are consistent to those reported here (48 $58 \%) .{ }^{23}$ No statistical differences were observed between the encapsulation efficiencies of the two microsphere groups reported here $(n=6)$.

The release kinetics of RA were performed in triplicate and analyzed over 28 days [Fig. 2 (G)]. Unloaded microspheres are omitted as there is no RA release to measure over 28 days. 4 $\mu \mathrm{g} / \mathrm{mg}$ microspheres showed an initial burst release followed by a period of gradual release while $30 \mu \mathrm{g} / \mathrm{mg}$ microspheres maintained a higher rate of release throughout the 28 day period. The 30 $\mu \mathrm{g} / \mathrm{mg}$ microspheres released a larger percentage of the encapsulated RA by day 28 than the 4 $\mu \mathrm{g} / \mathrm{mg}$ microspheres suggesting that release rates are influenced by the amount of RA encapsulated in the microspheres. After 28 days, the $4 \mu \mathrm{g} / \mathrm{mg}$ microspheres had a cumulative RA release of $28 \pm 10 \%$, and the $30 \mu \mathrm{g} / \mathrm{mg}$ microspheres had a cumulative RA release of $53 \pm 2 \%$ of the initial encapsulation. The cumulative release percent between the two groups remained statistically similar until day 24 .

\section{Aggregate formation and microsphere incorporation}

Murine ESC aggregates were used to estimate an incorporation ratio of microspheres to cells [Supplemental Fig. 1 (A)]. It was found that $0.5 \mathrm{mg}$ of microspheres did not provide an even distribution of microspheres in all mESC aggregates but many mESC aggregates did contain microspheres after 24 hours [Supplemental Fig. $1\left(\mathrm{~A}_{\mathrm{IIb}}\right)$ ]. The microspheres used with mESC aggregates had an expected microsphere diameter of $20.01 \pm 13.64 \mu \mathrm{m}$ [Supplemental Fig. $\left.1\left(\mathrm{~B}_{\mathrm{I}}, \mathrm{B}_{\mathrm{II}}\right)\right]$. This large diameter is likely to have a detrimental effect on microsphere incorporation and may cause a higher RA rate of release than desired due to the surface area to volume ratio [Supplemental Fig. $1\left(\mathrm{~B}_{\mathrm{III}}\right)$ ]. Both of these issues were addressed by making smaller 
4 and $30 \mu \mathrm{g} / \mathrm{mg}$ microspheres for our studies with hiPSC aggregates. However, the mESC microsphere experiment did provide critical insight for the incorporation of RA releasing microspheres in hiPSC aggregates. Specifically, the mESC aggregates that contained microspheres remained intact after removal from the microwells [Supplemental Fig. $1\left(\mathrm{~A}_{\text {IIIb }}\right)$. Viability studies performed on day 8 showed that RA releasing PCL microspheres did not result in localized cell death [Supplemental Fig. $1\left(\mathrm{C}_{\mathrm{I}-\mathrm{VI}}\right)$ ]. Flow cytometry performed on day 8 also confirmed a decrease in expression of pluripotency markers (SOX2 and SSEA-1) by mESC aggregates that contained microspheres [Supplemental Fig. $1\left(\mathrm{C}_{\mathrm{VII}}, \mathrm{C}_{\mathrm{VIII}}\right)$ ].

In an effort to increase the amount of microspheres per cells, $3 \mathrm{mg}$ of $30 \mu \mathrm{g} / \mathrm{mg}$ microspheres were incorporated into hiPSC aggregates. However, this amount turned out to be detrimental for aggregate formation as it covered the cells completely [Supplemental Fig. 2 (C)]. After 5 days of culture, most of the hiPSCs aggregates tested with $3 \mathrm{mg}$ of microspheres disintegrated [Supplemental Fig. 2 (D)]. Based on these preliminary studies, $0.5 \mathrm{mg}$ of microspheres were incorporated in hiPSC aggregates. hiPSCs without microspheres formed spherical aggregates after 24 hours of culture in microwells [Fig. 3 (A, B)]. Microspheres appear as dark spheroids mixed with hiPSCs at Day 0 [Fig. 3 (C, E, G)]. After 24 hours, microspheres are engulfed by hiPSCs forming tight aggregates that maintained their integrity when transferred to PLO/laminin-coated plates on day 5 [Fig. 3 (D, F, H)].

\section{Flow cytometry}

Flow cytometry of hiPSC aggregates was performed on Day 5 of cell culture to assess cell viability and determine if the controlled release of RA from the microspheres had any effect on cell differentiation. After 5 days, the percent viability of hiPSC and hiPSC-microsphere 
aggregates remained similar to Day 0 undifferentiated cells [Fig. 4 (A)]. The viability studies showed that hiPSC aggregates were not negatively affected by sterilized PCL microspheres. Similarly, hiPSC-microsphere aggregates remained tightly aggregated after 5 days. This observation is encouraging as it implies that strong aggregate formation is sustainable with PCL microspheres and that the PCL itself is not leading to cell death. Expression of pluripotent marker SSEA-4 significantly decreased in hiPSC-microsphere aggregates containing RA releasing microspheres when compared to Day 0 undifferentiated cells [Fig. 4 (B)]. SSEA-4 expression of the negative control and unloaded microspheres groups remained similar to Day 0 levels. The controlled release of RA is likely to be inducing differentiation as the cells were more differentiated than the untreated negative control and unloaded microsphere groups.

\section{Morphology of differentiated cell cultures}

By day 5 of cell culture, hiPSC-microsphere aggregates remained spherically uniform and similar to both control groups without microspheres [Fig. 5 (A, E, I, M, Q)]. By Day 9 of cell culture, hiPSC-microsphere aggregates had readily adhered to PLO/laminin-coated surfaces revealing the location of many microspheres as dark spheroids located throughout the aggregates [Fig. $5(\mathrm{~J}, \mathrm{~N}, \mathrm{R})]$. Day 12 phase contrast imaging of cell culture confirmed that cell growth continued in all experimental groups and was not inhibited by the presence of PCL microspheres [Fig. 5 (C, G, K, O, S)]. Immunocytochemistry performed at Day 12 showed neurite outgrowth in hiPSC and hiPSC-microsphere aggregates indicative of early neuron differentiation [Fig. 5 (D, $\mathrm{H}, \mathrm{L}, \mathrm{P}, \mathrm{T})]$. Both control groups and experimental cultures expressed the neuronal marker TUJ1 in green and cell bodies were visualized with DAPI in blue. Quantitative analysis of TUJ1 positive images revealed that relative aggregate surface area is higher with the $30 \mu \mathrm{g} / \mathrm{mg}$ and lower with unloaded microspheres groups when compared to the untreated control [Fig. 6]. 
Additionally, relative neurite length and degree of branching is more pronounced in the hiPSC aggregates treated with soluble RA and with unloaded microspheres when compared to the untreated controls. The 4 and $30 \mu \mathrm{g} / \mathrm{mg}$ hiPSC-microspheres groups showed less branching and shorter neurites when compared to the untreated controls.

Further SEM analysis of Day 12 hiPSC-unloaded microspheres aggregates showed that the majority of incorporated microspheres were less than $10 \mu \mathrm{m}$ in diameter, congruent with previously published studies [Fig. 7 (A, B)]. ${ }^{10}$ Similarly, the microspheres are relegated to the interstitial space of the aggregate. Microspheres are false colored in light green and the cells are false colored in pink. SEM analysis of hiPSC aggregates showed little bulk degradation of PCL by day 12 [Fig. 7 (B)].

\section{Discussion and future work}

In this study, we demonstrated that PCL microsphere-mediated delivery of RA can overcome diffusion barriers of soluble morphogens into 3D hiPSC aggregates. PCL was chosen as an affordable alternative to other polymers and for its potential biocompatibility with cell culture. These features make PCL a suitable polymer for neural tissue engineering applications. A single emulsion technique was used to produce large quantities of microspheres in a relatively short period of time. The expected diameter analysis of the RA releasing microspheres reported here point towards a reproducible target size. Carpenedo et al. showed that microsphere diameter affects incorporation efficiency within mESC aggregates with smaller diameter microspheres (1 $\mu \mathrm{m})$ being more readily incorporated within mESC aggregates than larger ones $(3 \mu \mathrm{m}){ }^{10}$ Additionally, the study revealed that incorporated microspheres ranged in size from 2 to $5 \mu \mathrm{m}$ while larger diameter microspheres $(11 \mu \mathrm{m})$ were excluded. The microspheres used by 
Carpenedo et al. remained within the interstitial space of the aggregate, as opposed to being internalized by ESCs, suggesting that an optimum microsphere size for interstitial incorporation exists. Our SEM analysis of the hiPSC-microsphere aggregates shown revealed that incorporated microspheres are less than $10 \mu \mathrm{m}$ in diameter.

The first attempt at incorporating microspheres with hiPSC aggregates resulted in poor aggregate formation; $3 \mathrm{mg}$ of microspheres per AggreWell ${ }^{\mathrm{TM}}$ insert proved to be a disruptive amount of particles for cell-cell binding. A number of cell adhesion molecules (CAMs) have been identified as regulating human PSC survival, proliferation, and differentiation. ${ }^{27}$ In particular, E-cadherin mediated cell-cell interactions are important for cell survival and even partial disruption of these can induce PSC blebbing and apoptosis. The increased number of microspheres in the hiPSC - $3 \mathrm{mg}$ microsphere aggregate trial may have partially blocked Ecadherin or other CAMs; this partial blocking was not enough induce the widespread cell death that is common in dissociated hiPSCs. While hiPSC aggregates were able to form during the 5 days in the AggreWell ${ }^{\mathrm{TM}}$ microwells, with insufficient cell-cell binding, the aggregates could not maintain their integrity during transfer to laminin-coated plates and subsequently broke apart. When hiPSCs were cultured with $0.5 \mathrm{mg}$ of microspheres, these instabilities were not observed. However, Rho-association protein kinase (ROCK)-inhibitors have been shown to increase dissociated human PSC survival and may also enhance cell survival in aggregates containing higher quantities of microspheres. Future studies will seek to further optimize the microsphere to cell ratio to produce hiPSC aggregates while providing optimum morphogen delivery to enhance differentiation.

The encapsulation efficiencies of the low-cost PCL microspheres reported here ( 60\%), are consistent with PLGA microspheres following a similar fabrication. ${ }^{23}$ It is possible that 
during the four-hour solvent evaporation a RA concentration equilibrium between the solvent and water phases may have been reached that prevented further diffusion of RA out of the PCL solution. The release studies show that RA cumulative release percent remained similar until day 24 , at which point the higher concentration microspheres released more RA. The SEM analysis at day 12 of incorporated microspheres showed little bulk degradation suggesting that RA release is mostly driven by diffusion. However, it is not known at this time if a higher quantity of RA in microspheres increases the degradation rate of PCL or if, in a longer term release study, the microspheres will begin to break apart and cause a spike in the RA released. Longer release studies would provide further insight into the mechanisms of RA release from PCL and whether the remaining RA would continue to be released gradually as the PCL degrades.

Quantitative viability studies and pluripotency marker expression confirmed that PCL is not cytotoxic to hiPSC aggregates and that microsphere mediated RA delivery lowers pluripotency marker expression in hiPSCs. Specifically, viability analysis across the hiPSC experimental groups revealed that the relative percentage of viable cells following 5 days of culture with PCL microspheres remained similar to undifferentiated hiPSCs with and without RA delivery. This result is consistent with observations made in the mESC aggregates containing microspheres where the presence of PCL microspheres did not result in localized cell death. Similarly, relative pluripotency marker percent expression of SSEA-4 in undifferentiated hiPSCs versus day 5 confirmed that RA enhanced the differentiation of hiPSC aggregates when compared to unloaded or no microsphere control groups. Moreover, day 12 immunocytochemistry revealed that all hiPSC aggregate groups were positive for TUJ1 and all groups successfully acquired neuronal morphology indicative of NPCs with no negative effects associated with PCL microsphere incorporation. hiPSCs can be restricted to ectoderm lineages 
within 12 days but further studies are required to determine the extent of the differentiation reported here. ${ }^{18}$ It is not known at this time if the hiPSCs will eventually express motor neuron markers, as the experiments did not extend past 12 days. While TUJ1 expression is an early neuronal marker, it cannot be used to determine what type of neurons are being produced, which would require further analysis.

Differences in cell morphology were observed for aggregates at day 12 with IncuCyte immunofluorescence assays. The aggregates that contained RA-releasing microspheres extended less neurites with fewer branches than the negative control while the unloaded microsphere sample extended neurite lengths and branch points similar to the positive control. One reason for this observed difference might be due to the fact that the neural aggregates in the $30 \mu \mathrm{g} / \mathrm{mg}$ sample group had a significantly greater spread than controls and other samples, making it harder to visualize the neurites. While the reason for this was not explored in this study, it is a distinct morphological difference. Additionally, samples were comprised of approximately 15 aggregates per well and the proximity of two aggregates near to each other may have limited the potential for neurite outgrowth. Previous studies have found that elevated concentrations of RA may be toxic. ${ }^{15}$ While there was no observable decrease in viability of these cells compared to the controls, a locally high concentration of RA within the aggregate may have altered gene expression in RA-microsphere-containing aggregates. Future studies of single aggregates per well would remove this variable and give enhanced insight into their morphology. RA gradients are known to be important for cell patterning in the developing nervous system and have been implicated in increasing proliferation in neural precursors. ${ }^{30}$ Similarly, the presence of RAreleasing microspheres may have contributed to a local gradient extending from the cell aggregate, increasing the EB area observed. Future studies should seek to optimize the RA 
concentration within microspheres to maximize neurite growth; ideally a lower concentration of RA locally delivered through microspheres would induce neurite growth comparable to soluble RA without the risk of toxicity.

Directed delivery of morphogens through microspheres is an efficient alternative to soluble delivery for the directed differentiation of hiPSC aggregates. Here, microspheres were fabricated in large numbers with a low-cost polymer, PCL, by a relatively simple single emulsion technique. The probability density estimate of microsphere size distributions suggests a very reproducible microsphere target size can be achieved. As discussed in previous studies, interstitial incorporation of microspheres is strongly favored in the $2-5 \mu \mathrm{m}$ range and our SEM analysis showed that our experiments were consistent with those results. Specifically, the microspheres located in the interstitial sections of fixed hiPSC aggregates had diameters less than $10 \mu \mathrm{m}$. Microspheres larger than $10 \mu \mathrm{m}$ are not well incorporated into PSC aggregates (observed in mESC aggregates) but remained on the aggregate surface and were easily lost during media changes or cell plating. Large quantities of microspheres tended to aggregate in the cell media instead of incorporating into PSC aggregates (observed with the $3 \mathrm{mg}$ microsphere hiPSC aggregate trial). However, successful incorporation of $0.5 \mathrm{mg}$ of microspheres within hiPSC aggregates resulted in RA delivery to regions otherwise difficult to access with traditional culture techniques, suggesting that microsphere to cell ratio can be further refined for positive effects in hiPSC aggregates.

Taken together, this work provides a foundation for further exploration of PCL microspheres for delivery of biologically active molecules. The results serve as a starting point for further experiments incorporating microspheres into PSC aggregates to direct neuronal differentiation of hiPSCs. The protocols for incorporating PCL microspheres within hiPSC 
aggregates reported in this study could be further refined to accommodate the fragile nature of hiPSC aggregates and to increase the incorporation rate of microspheres. Finally, other small molecules that influence stem cell differentiation into neural lineages, such as purmorphamine, are promising candidates for encapsulation into PCL to induce differentiation into motor neurons or other neuronal cell-types. ${ }^{22,24,28}$

\section{Conflicts of Interest}

Jose Carlos Gomez, John M. Edgar, Andrew M. Agbay, Emma Bibault, Amy Montgomery, Nima Khadem Mohtaram, and Stephanie M. Willerth have no conflicts of interest to declare.

\section{Ethical Standards}

No human subjects or animals were used in these studies. All human pluripotent stem cell work was conducted according to the CIHR's Stem Cell Oversight Committee guidelines and with approval with the Human Research Ethics Board at the University of Victoria.

\section{Acknowledgements}

The authors would like to thank funding from the University of Victoria, the Canada Research Chairs program, the Rick Hansen Foundation, and the Natural Science and Engineering Research Council for funding for this work. 


\section{References}

1. Agbay, A., Mohtaram, N. and Willerth, S. Controlled release of glial cell line-derived neurotrophic factor from poly(E-caprolactone) microspheres. Drug Delivery and Translational Research. 4: 159-170, 2014.

2. Anderson, J. M. and Shive, M. S. Biodegradation and biocompatibility of PLA and PLGA microspheres. Adv. Drug Del. Rev. 64, Supplement: 72-82, 2012.

3. Andrews, P. W. From teratocarcinomas to embryonic stem cells. Philosophical Transactions of the Royal Society of London Series B-Biological Sciences. 357: 405-417, 2002.

4. Bain, G. and Gottlieb, D. I. Expression of Retinoid-X Receptors in P19 Embryonal Carcinoma-Cells and Embryonic Stem-Cells. Biochem. Biophys. Res. Commun. 200: 1252-1256, 1994.

5. Baker, M. Embryoid bodies get organized. Nature Reports Stem Cells 2008.

6. Ben-David, U. and Benvenisty, N. The tumorigenicity of human embryonic and induced pluripotent stem cells. Nat. Rev. Cancer. 11: 268-277, 2011.

7. Bratt-Leal, A. M., Carpenedo, R. L., Ungrin, M. D., Zandstra, P. W. and McDevitt, T. C. Incorporation of biomaterials in multicellular aggregates modulates pluripotent stem cell differentiation. Biomaterials. 32: 48-56, 2011.

8. Bratt-Leal, A. M., Nguyen, A. H., Hammersmith, K. A., Singh, A. and McDevitt, T. C. A microparticle approach to morphogen delivery within pluripotent stem cell aggregates. Biomaterials. 34: 7227-7235, 2013.

9. $\quad$ Carpenedo, R. L., Bratt-Leal, A. M., Marklein, R. A., Seaman, S. A., Bowen, N. J., McDonald, J. F. and McDevitt, T. C. Homogeneous and organized differentiation within embryoid bodies induced by microsphere-mediated delivery of small molecules. Biomaterials. 30: 2507-2515, 2009.

10. Carpenedo, R. L., Seaman, S. A. and McDevitt, T. C. Microsphere size effects on embryoid body incorporation and embryonic stem cell differentiation. Journal of Biomedical Materials Research Part A. 94A: 466-475, 2010.

11. Cyranoski, D. Japanese woman is first recipient of next-generation stem cells. Nature, 2014.

12. Damjanov, I. and Andrews, P. W. The terminology of teratocarcinomas and teratomas. Nat Biotech. 25: 1212-1212, 2007.

13. Dang, S. M., Kyba, M., Perlingeiro, R., Daley, G. Q. and Zandstra, P. W. Efficiency of embryoid body formation and hematopoietic development from embryonic stem cells in different culture systems. Biotechnol. Bioeng. 78: 442-453, 2002.

14. de Lazaro, I., Yilmazer, A. and Kostarelos, K. Induced pluripotent stem (iPS) cells: A new source for cellbased therapeutics? J. Controlled Release. 185: 37-44, 2014.

15. Duester, G. Retinoic Acid Synthesis and Signaling during Early Organogenesis. Cell. 134: 921-931,

16. Evans, M. J. and Kaufman, M. H. Establishment in culture of pluripotential cells from mouse embryos. Nature. 292: 154-156, 1981.

17. Hackett, Jamie A. and Surani, M. A. Regulatory Principles of Pluripotency: From the Ground State Up. Cell Stem Cell. 15: 416-430, 2014.

18. Haile, Y., Nakhaei-Nejad, M., Boakye, P. A., Baker, G., Smith, P. A., Murray, A. G., et al. Jahroudi, N. Reprogramming of HUVECs into Induced Pluripotent Stem Cells (HiPSCs), Generation and Characterization of HiPSC-Derived Neurons and Astrocytes. PLoS One. 10, 2015.

19. Hines, D. J., Hines, R. M., Mulligan, S. J. and Macvicar, B. A. Microglia processes block the spread of damage in the brain and require functional chloride channels. Glia. 57: 1610-1618, 2009.

20. Hofstetter, C. P., Holmstrom, N. A. V., Lilja, J. A., Schweinhardt, P., Hao, J., Spenger, C., et al. Olson, L. Allodynia limits the usefulness of intraspinal neural stem cell grafts; directed differentiation improves outcome. Nat. Neurosci. 8: 346-353, 2005.

21. Hu, B. Y., Weick, J. P., Yu, J. Y., Ma, L. X., Zhang, X. Q., Thomson, J. A. and Zhang, S. C. Neural differentiation of human induced pluripotent stem cells follows developmental principles but with variable potency. Proceedings of the National Academy of Sciences of the United States of America. 107: 43354340, 2010.

22. Hu, B. Y. and Zhang, C. Differentiation of spinal motor neurons from pluripotent human stem cells. Nature Protocols. 4: 1295-1304, 2009.

23. Jeong, Y.-I., Song, J.-G., Kang, S.-S., Ryu, H.-H., Lee, Y.-H., Choi, C., et al. Jung, S. Preparation of poly(DL-lactide-co-glycolide) microspheres encapsulating all-trans retinoic acid. International Journal of Pharmaceutics (Kidlington). 259: 79-91, 2003. 
24. Karumbayaram, S., Novitch, B. G., Patterson, M., Umbach, J. A., Richter, L., Lindgren, A., et al. Lowry, W. E. Directed Differentiation of Human-Induced Pluripotent Stem Cells Generates Active Motor Neurons. Stem Cells. 27: 806-811, 2009.

25. Kinney, M. A., Hookway, T. A., Wang, Y. and McDevitt, T. C. Engineering Three-Dimensional Stem Cell Morphogenesis for the Development of Tissue Models and Scalable Regenerative Therapeutics. Ann. Biomed. Eng. 42: 352-367, 2014.

26. Koichi Hayashi, Masayuki Hashimoto, Masao Koda, Atsuhiko T. Naito, Atsushi Murata, Akihiko Okawa, et al. Masashi Yamazaki. Increase of sensitivity to mechanical stimulus after transplantation of murine induced pluripotent stem cell-derived astrocytes in a rat spinal cord injury model. Journal of Neurosurgery: Spine. 15: 582-593, 2011.

27. Li, L., Bennett, S. A. L. and Wang, L. S. Role of E-cadherin and other cell adhesion molecules in survival and differentiation of human pluripotent stem cells. Celll Adhes. Migr. 6: 59-70, 2012.

28. Li, X. J., Hu, B. Y., Jones, S. A., Zhang, Y. S., Lavaute, T., Du, Z. W. and Zhang, S. C. Directed differentiation of ventral spinal progenitors and motor neurons from human embryonic stem cells by small molecules. Stem Cells. 26: 886-893, 2008.

29. Lopez-Gonzalez, R. and Velasco, I. Therapeutic Potential of Motor Neurons Differentiated from Embryonic Stem Cells and Induced Pluripotent Stem Cells. Archives of Medical Research. 43: 1-10, 2012.

30. Maden, M. Retinoic acid in the development, regeneration and maintenance of the nervous system. Nat. Rev. Neurosci. 8: 755-765, 2007.

31. Mohtaram, N. K., Ko, J., Montgomery, A., Carlson, M., Sun, L., Wong, A., et al. Willerth, S. M. Multifunctional Electrospun Scaffolds for Promoting Neuronal Differentiation of Induced Pluripotent Stem Cells. Journal of Biomaterials and Tissue Engineering. 4: 906-914, 2014.

32. Montgomery, A., Wong, A., Gabers, N. and Willerth, S. M. Engineering personalized neural tissue by combining induced pluripotent stem cells with fibrin scaffolds. Biomaterials Science, 2015.

33. Mothe, A. J. and Tator, C. H. Review of transplantation of neural stem/progenitor cells for spinal cord injury. Int. J. Dev. Neurosci. 31: 701-713, 2013.

34. Novitch, B. G., Wichterle, H., Jessell, T. M. and Sockanathan, S. A requirement for retinoic acid-mediated transcriptional activation in ventral neural patterning and motor neuron specification. Neuron. 40: 81-95, 2003.

35. Rhinn, M. and Dolle, P. Retinoic acid signalling during development. Development. 139: 843-858, 2012.

36. Rungarunlert, S., Techakumphu, M., Pirity, M. K. and Dinnyes, A. Embryoid body formation from embryonic and induced pluripotent stem cells: Benefits of bioreactors. World journal of stem cells. 1: 1121, 2009.

37. Sachlos, E. and Auguste, D. T. Embryoid body morphology influences diffusive transport of inductive biochemicals: A strategy for stem cell differentiation. Biomaterials. 29: 4471-4480, 2008.

38. Salimi, A., Nadri, S., Ghollasi, M., Khajeh, K. and Soleimani, M. Comparison of different protocols for neural differentiation of human induced pluripotent stem cells. Mol. Biol. Rep. 41: 1713-1721, 2014.

39. Tabar, V. and Studer, L. Pluripotent stem cells in regenerative medicine: challenges and recent progress. Nat. Rev. Genet. 15: 82-92, 2014.

40. Takahashi, K., Tanabe, K., Ohnuki, M., Narita, M., Ichisaka, T., Tomoda, K. and Yamanaka, S. Induction of pluripotent stem cells from adult human fibroblasts by defined factors. Cell. 131: 861-872, 2007.

41. Takahashi, K. and Yamanaka, S. Induction of pluripotent stem cells from mouse embryonic and adult fibroblast cultures by defined factors. Cell. 126: 663-676, 2006.

42. Thomson, J. A., Itskovitz-Eldor, J., Shapiro, S. S., Waknitz, M. A., Swiergiel, J. J., Marshall, V. S. and Jones, J. M. Embryonic stem cell lines derived from human blastocysts. Science. 282: 1145-1147, 1998.

43. Toyooka, Y., Tsunekawa, N., Akasu, R. and Noce, T. Embryonic stem cells can form germ cells in vitro. Proceedings of the National Academy of Sciences of the United States of America. 100: 11457-11462, 2003.

44. Van Winkle, A. P., Gates, I. D. and Kallos, M. S. Mass Transfer Limitations in Embryoid Bodies during Human Embryonic Stem Cell Differentiation. Cells Tissues Organs. 196: 34-47, 2012.

45. Vroemen, M., Aigner, L., Winkler, J. and Weidner, N. Adult neural progenitor cell grafts survive after acute spinal cord injury and integrate along axonal pathways. Eur. J. Neurosci. 18: 743-751, 2003.

46. Wang, A., Tang, Z., Park, I.-H., Zhu, Y., Patel, S., Daley, G. Q. and Li, S. Induced pluripotent stem cells for neural tissue engineering. Biomaterials. 32: 5023-5032, 2011.

47. Willerth, S. M. Neural tissue engineering using embryonic and induced pluripotent stem cells. Stem Cell Research \& Therapy. 2, 2011. 
48. Willerth, S. M., Faxel, T. E., Gottlieb, D. I. and Sakiyama-Elbert, S. E. The effects of soluble growth factors on embryonic stem cell differentiation inside of fibrin scaffolds. Stem Cells. 25: 2235-2244, 2007.

49. Willerth, S. M. and Sakiyama-Elbert, S. E. Cell therapy for spinal cord regeneration. Adv. Drug Del. Rev. 60: 263-276, 2008.

50. Wischke, C. and Schwendeman, S. P. Principles of encapsulating hydrophobic drugs in PLA/PLGA microparticles. Int. J. Pharm. 364: 298-327, 2008.

51. Woodruff, M. A. and Hutmacher, D. W. The return of a forgotten polymer-Polycaprolactone in the 21st century. Progress in Polymer Science. 35: 1217-1256, 2010.

52. Xie, J. W., Willerth, S. M., Li, X. R., Macewan, M. R., Rader, A., Sakiyama-Elbert, S. E. and Xia, Y. N. The differentiation of embryonic stem cells seeded on electrospun nanofibers into neural lineages. Biomaterials. 30: 354-362, 2009.

53. Zhou, X., He, X. and Ren, Y. Function of microglia and macrophages in secondary damage after spinal cord injury. Neural Regeneration Research. 9: 1787-1795, 2014. 


\begin{tabular}{|c|c|}
\hline RA Concentration Added & Encapsulation Efficiency (\%) \\
\hline $4 \mu \mathrm{g} / \mathrm{mg}$ RA/PCL & $60.9 \pm 1.9$ \\
\hline $30 \mu \mathrm{g} / \mathrm{mg} \mathrm{RA} / \mathrm{PCL}$ & $58.4 \pm 3.3$ \\
\hline
\end{tabular}

Table 1. Encapsulation efficiencies for various drug loadings. ( $n=6$, mean \pm standard deviation) 
Figure 1. Schematic of retinoic acid releasing microspheres incorporated with pluripotent stem cell aggregates for promoting neuronal differentiation.

Figure 2. Scanning electron microscopy and characterization of unloaded, $4 \mu \mathrm{g} / \mathrm{mg}$, and 30 $\mu \mathrm{g} / \mathrm{mg}$ microspheres incorporated in human induced pluripotent stem cell aggregates. (A - C) Scanning electron micrographs showing some clustering and overall smooth morphology. (D - F) Histogram and probability density function estimate showing expected particle diameters. Sample size $\mathrm{n}=100$. (G) Cumulative release of all-trans retinoic acid over 28 days of $4 \mu \mathrm{g} / \mathrm{mg}$ compared to $30 \mu \mathrm{g} / \mathrm{mg}$. Sample size $\mathrm{n}=3$. * indicates $\mathrm{p}<0.05$ per day.

Figure 3. Phase contrast imaging at 10X magnification of human induced pluripotent stem cell aggregates. (A, B) Aggregate formation without microspheres initially and after 24 hours. (C, D) Aggregate formation with microspheres; unloaded microspheres appear as dark spheroid objects mixed with human induced pluripotent stem cells. (E, F) Aggregate formation with $4 \mu \mathrm{g} / \mathrm{mg}$ RA/PCL microspheres initially and after 24 hours. (G, H) Aggregate formation with $30 \mu \mathrm{g} / \mathrm{mg}$ microspheres initially and after 24 hours; 4 and $30 \mu \mathrm{g} / \mathrm{mg}$ microspheres become incorporated with human induced pluripotent stem cells after 24 hours.

Figure 4. Quantitative viability results and SSEA-4 expression for human induced pluripotent stem cell aggregates initially and after microsphere incorporation following 5 days of culture. (A) Percentage of viable cells measured by flow cytometry for experimental groups at Day 0 , and Day 5. No significant differences were measured between Day 0 cells and Day 5 groups. Sample size $n=3$. (B) Expression of stage-specific embryonic antigen-4 pluripotency marker at Day 0 and Day 5 of culture. Retinoic acid releasing microsphere groups expressed lower levels of marker after a 5 day culture period. Sample size $n=3$. * indicates $p<0.05$. 
Figure 5. Timeline of microsphere effects on human induced pluripotent stem cell aggregates following a neural induction differentiation protocol. (A, E, I, M, Q) Phase contrast imaging 10X magnification of intact aggregates following plate transfer after 5 days of cell culture. (B, F, J, N, R) Day 9 of cell culture shows that all aggregates have adhered to PLO/Laminin coated plates with microspheres shown as dark spheroids in the experimental groups. (C-D, G-H, K-L, O-P, ST) Day 12 of cell culture shows that all aggregates have extended neurites; Positive control groups expressed similar neurite morphology (TUJ1 - green) to the experimental groups in contrast with the negative control (no RA).

Figure 6. Quantitative analysis of hiPSC-aggregate morphology. The white bars represent relative neurite length per aggregate. The dark grey bar represents relative neurite branching per aggregate. The light grey represents relative surface area per aggregate. Untreated aggregates have a sample size of $n=30$. Soluble RA aggregates have a sample size of $n=21$. Unloaded aggregates have sample size of $n=27.4 \mu \mathrm{g} / \mathrm{mg}$ aggregates have a sample size of $\mathrm{n}=28.30$ $\mu \mathrm{g} / \mathrm{mg}$ aggregates have a sample size of $\mathrm{n}=32$.

Figure 7. False color scanning electron micrograph of unloaded polycaprolactone microspheres incorporated in human induced pluripotent stem cell aggregates. (A) Cell bodies are represented in pink while microspheres are represented in light green. (B) Microspheres are located outside cell bodies and show some clustering and surface degradation.

Supplemental 1. Murine embryonic stem cell aggregates tested with $0.5 \mathrm{mg}$ of microspheres. (A) Timeline for murine embryonic stem cell aggregates with incorporated microspheres after 25 days of culture. (B) Scanning electron microscopy and characterization of $3 \mu \mathrm{g} / \mathrm{mg}$ microspheres used with murine embryonic stem cell aggregates. Expected microsphere diameter sample size $\mathrm{n}$ 
$=103$. Percentage of all-trans retinoic acid cumulative release has a sample size of $n=2$. $(C)$ Viability studies and pluripotency marker expression analysis of murine embryonic stem cell aggregates following 8 days of culture. Viability and marker expression have a sample size of $n$ $=2$.

Supplemental 2. Phase contrast imaging 10X magnification of human induced pluripotent stem cell aggregates tested with $3 \mathrm{mg}$ of microspheres. (A, B) Untreated human induced pluripotent stem cells form tight aggregates after 24 hours and readily adhere to PLO/Laminin coated plates. (C, D) Microsphere treated human induced pluripotent stem cells are completely covered by microspheres after 24 hours and then aggregates disintegrate during plate transfer.

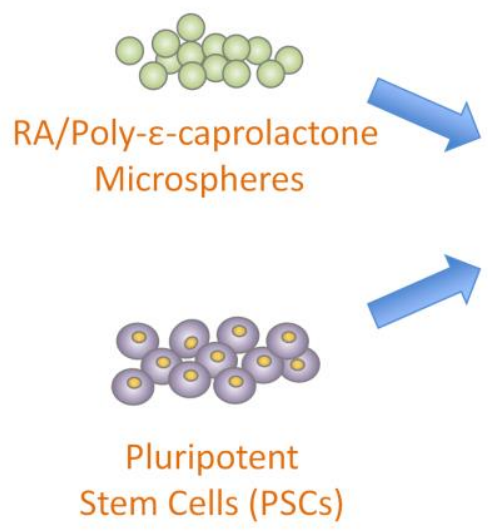

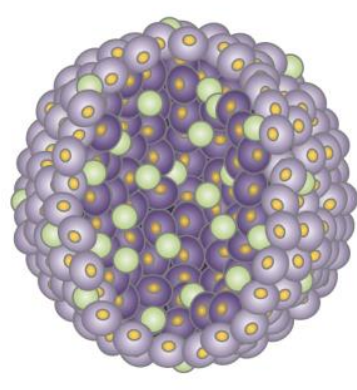

PSC Aggregate (Embryoid Body)

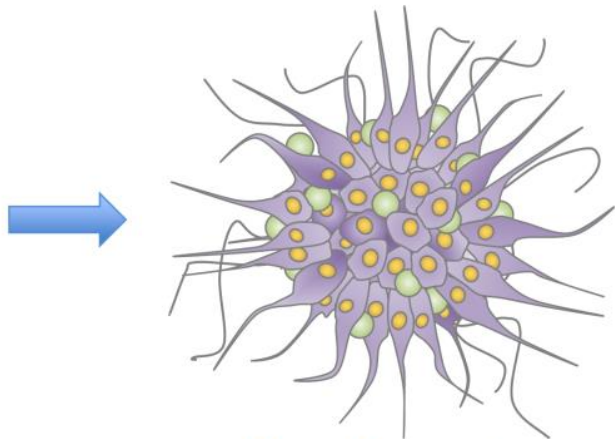

Neural Aggregate 

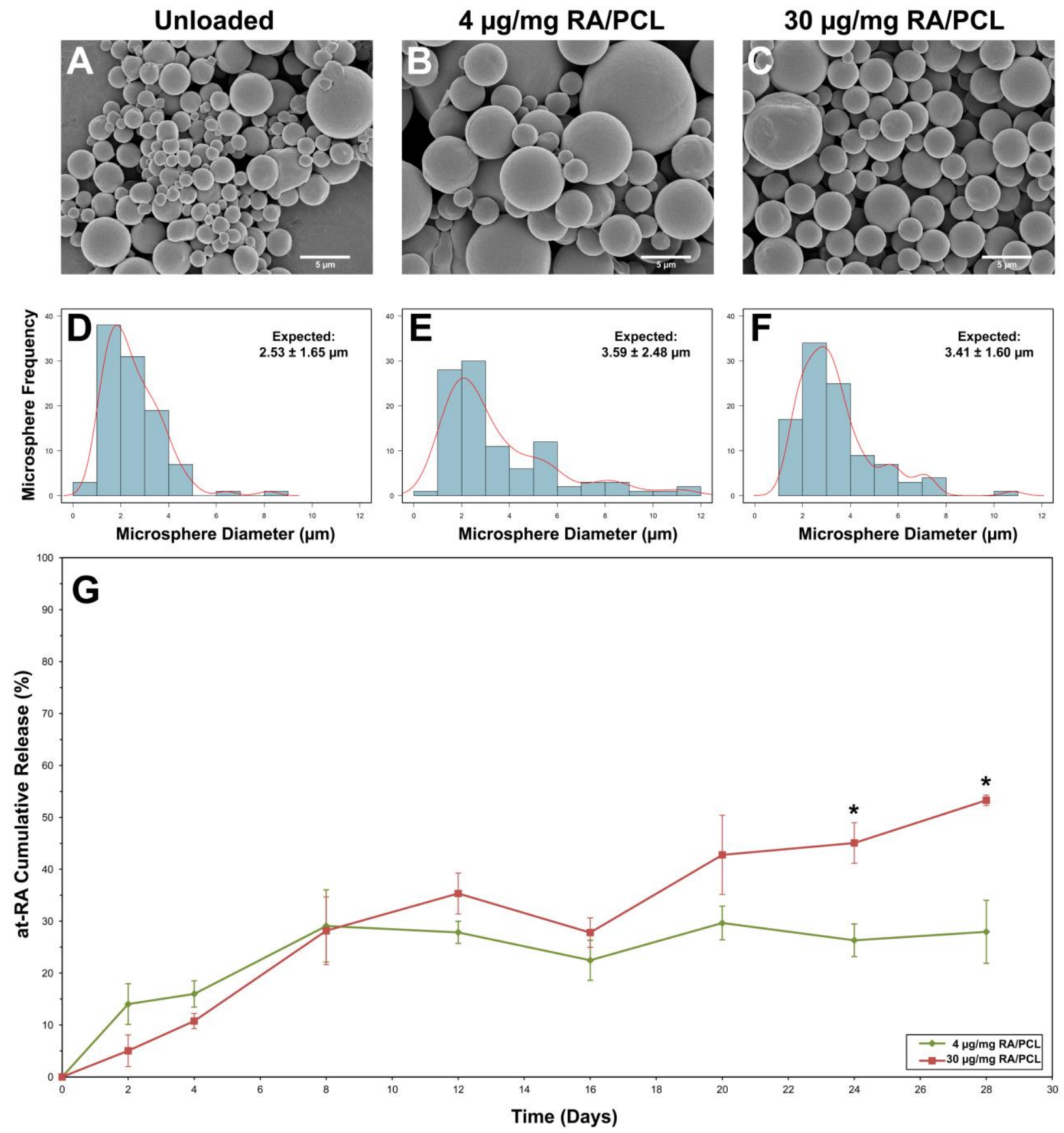

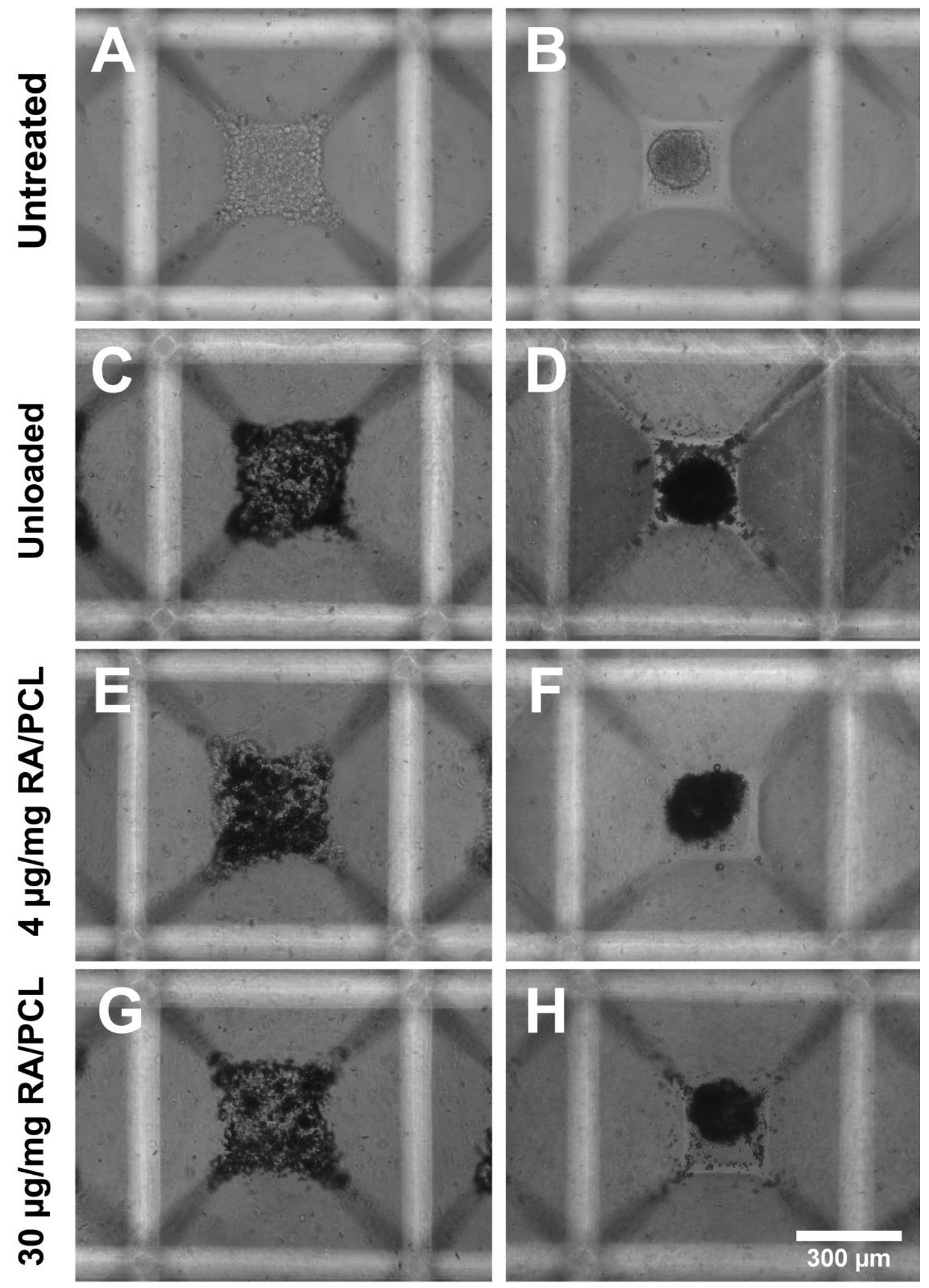

Day 0

\section{Day 1}



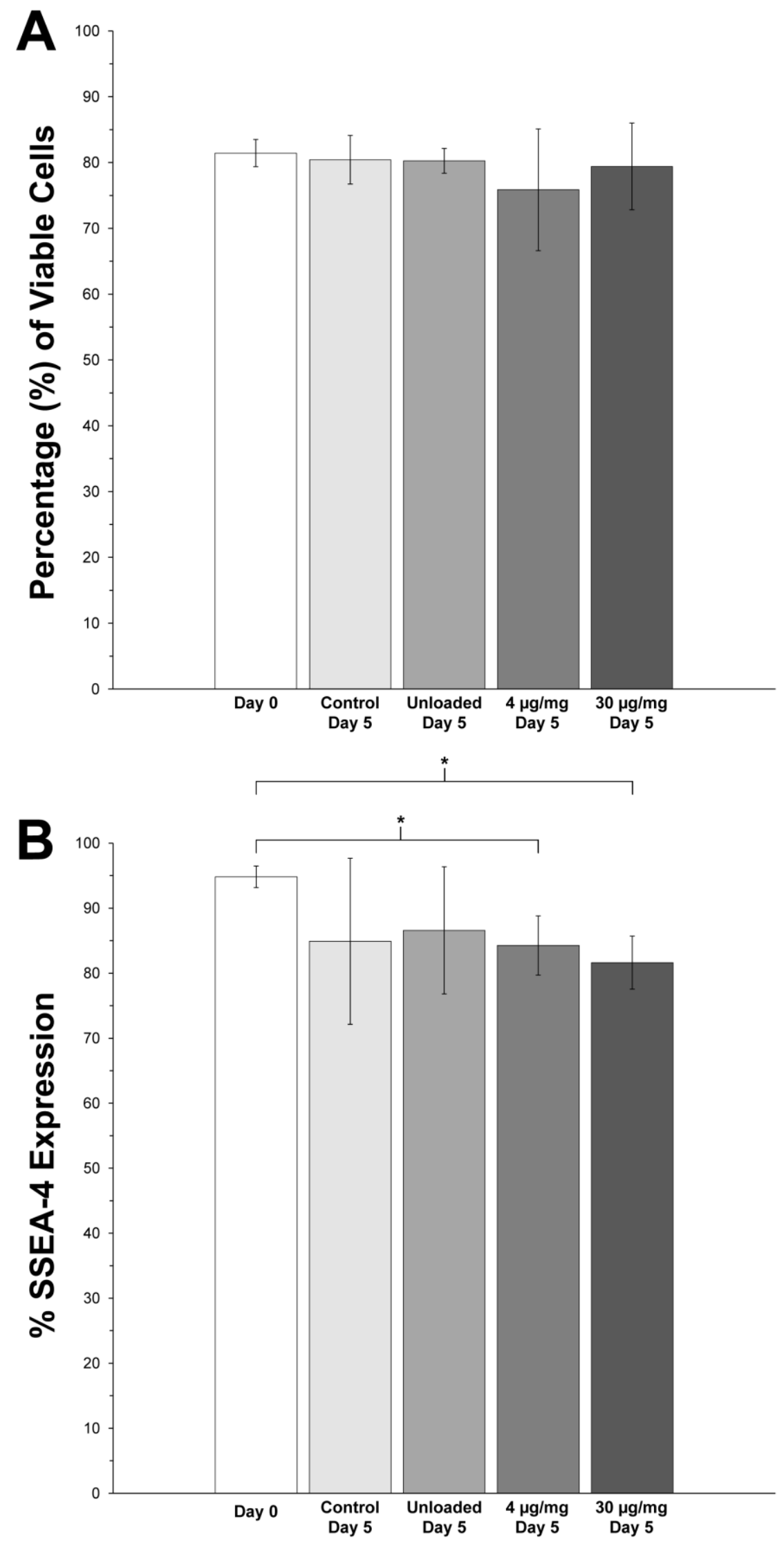

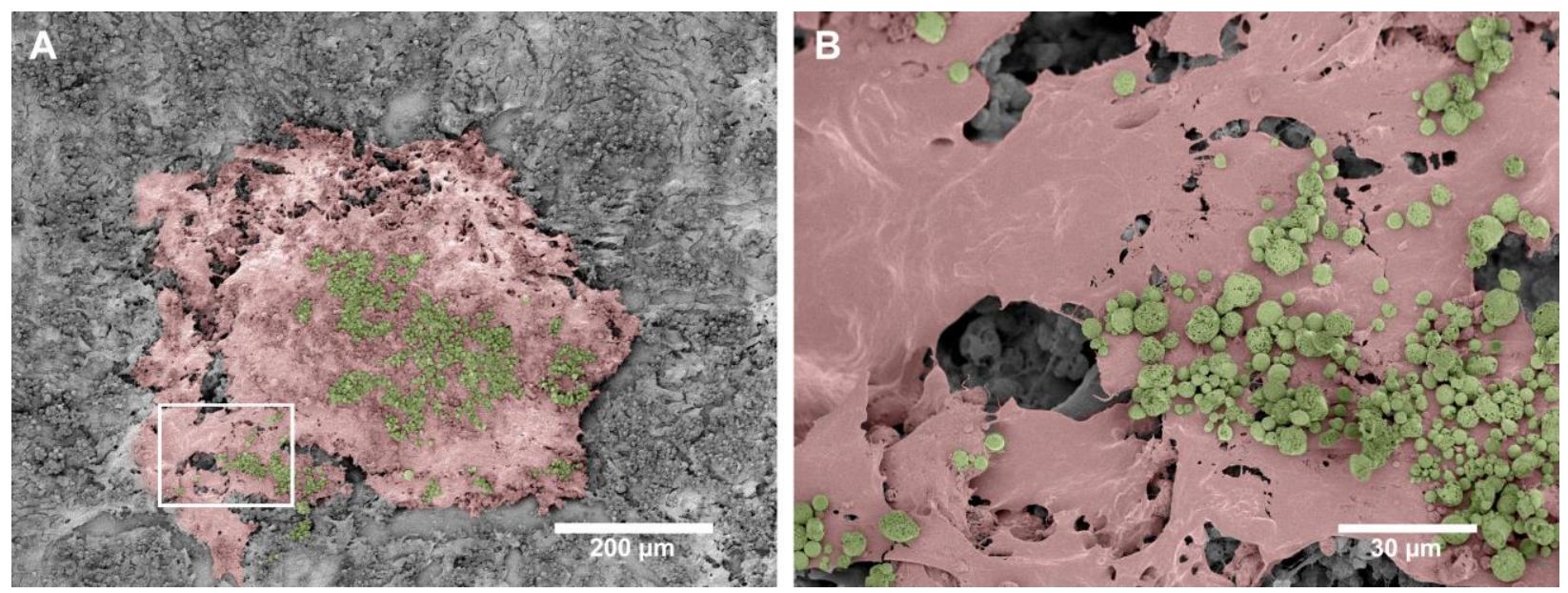

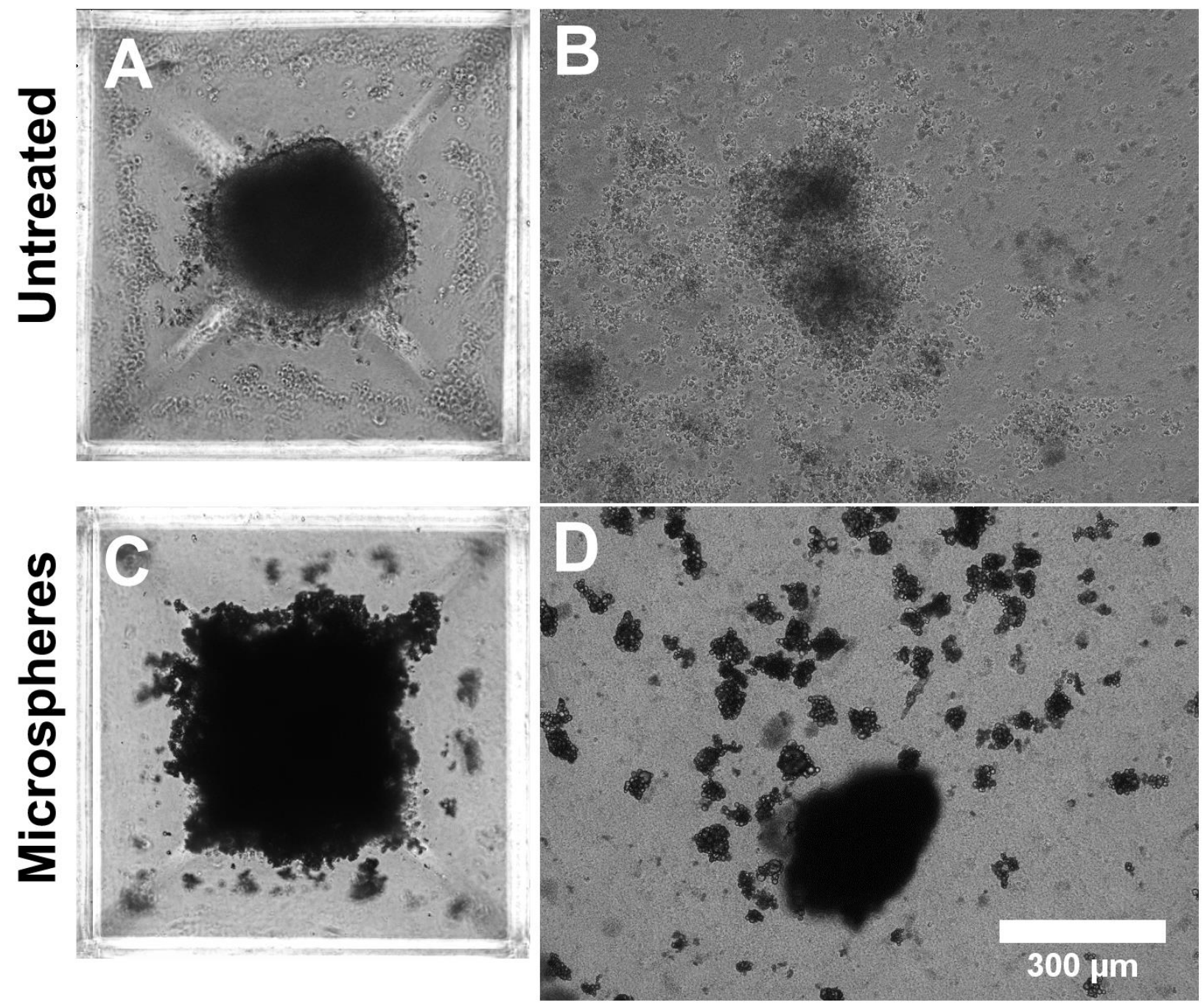

Day 1

Day 5 


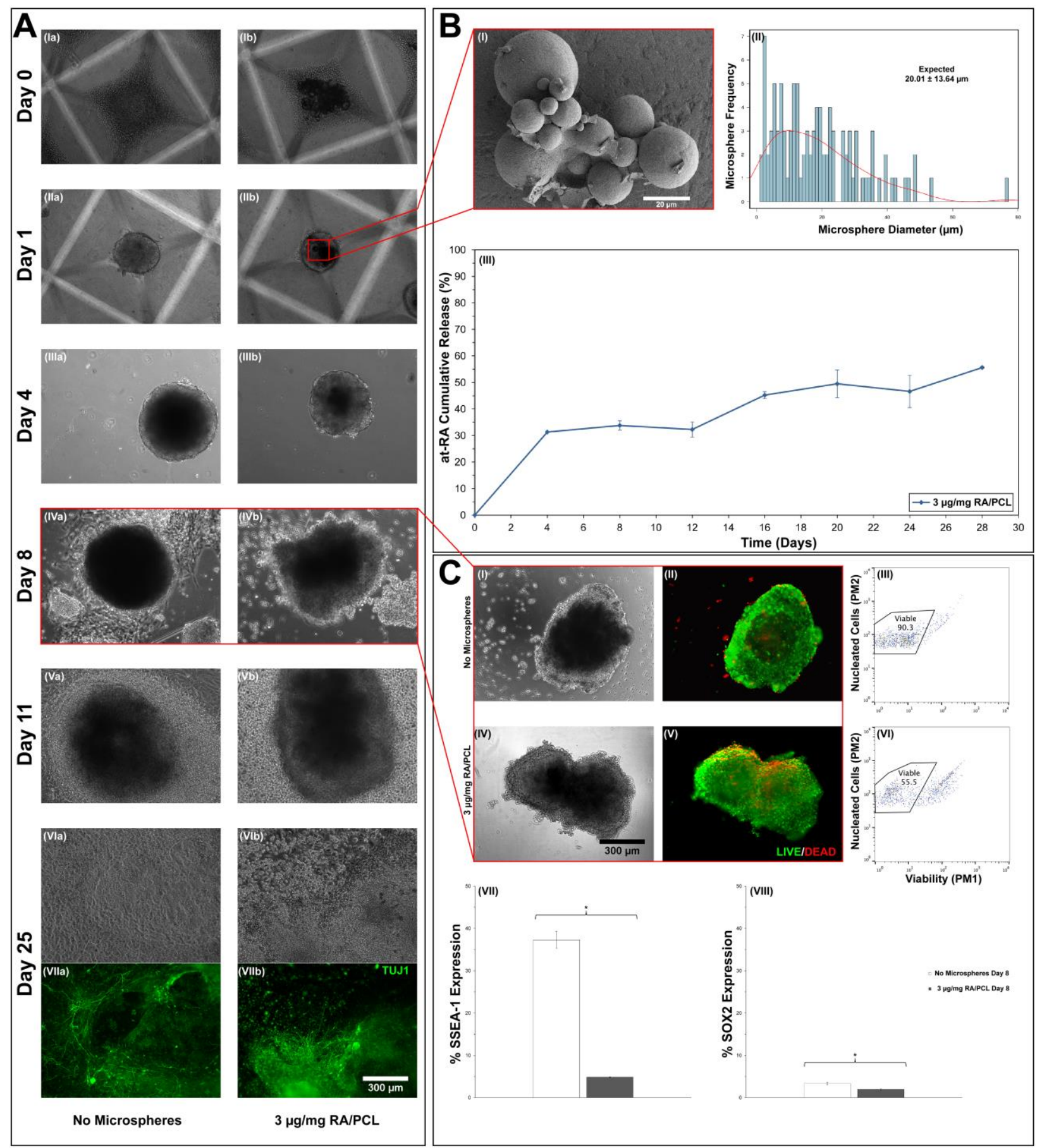


